\title{
HIGHER INTEGRABILITY AND THE BOUNDARY DIMENSION
}

\author{
Bruce Hanson and Pekka Koskela
}

\begin{abstract}
We answer a question posed by K. Astala and P. Koskela in the negative by producing examples for each $n \geq 2$ and each $K>1$ of bounded domains $D, D^{\prime}$ in $\mathbf{R}^{n}$ quasiconformally equivalent to the unit ball of $\mathbf{R}^{n}$ and a $K$-quasiconformal mapping $f$ of $D$ onto $D^{\prime}$ such that $f$ lies in $\operatorname{locLip}_{\alpha}(D)$ for all $0<\alpha<1$ and $\operatorname{dim}_{H}(\partial D)=\operatorname{dim}_{H}\left(\partial D^{\prime}\right)=n-1$ but the derivative of of $f$ does not belong to $L^{p}(D)$ for any exponent $p>n$.
\end{abstract}

\section{Introduction}

Let $D, D^{\prime}$ be domains in $R^{n}$ with $D^{\prime}$ bounded and let $f$ be a $K$-quasiconformal mapping of $D$ onto $D^{\prime}$. Then, as is well-known, $f$ is differentiable a.e. on $D$ and $\int_{D}\left|f^{\prime}\right|^{n} d m<\infty$; in fact, $\int_{D}\left|f^{\prime}\right|^{n} d m \leq K|f(D)|$ (where $|S|$ denotes the $n$-measure of $S \subset R^{n}$ ). On the other hand, it need not be true that

$$
\int_{D}\left|f^{\prime}\right|^{p} d m<\infty \quad \text { for some } \quad p>n .
$$

Astala and Koskela [AK] recently showed that the higher integrability (1) is closely connected with the local Hölder continuity properties of $f$ on $D$. They point out that (1) implies

$$
f \in \operatorname{locLip}_{\alpha}(D) \quad \text { for some } \quad 0<\alpha \leq 1
$$

Moreover, they show that the converse is true if one assumes that $\partial D^{\prime}$ is not "too thick" in the sense of the Minkowski dimension. They also ask whether the converse remains true without the additional assumption on $\partial D^{\prime}$. The main purpose of this note is to give an example which shows that some assumption of this type is necessary. Before stating our results, we define some of the terms used above and state Astala and Koskela's result as well.

The research for this paper was done while the first author was visiting at the University of Uppsala in Sweden and the second author at the Institut Mittag-Leffler. They wish to thank the institutions for their hospitality and support.

AMS Subject Classification: Primary 30C65. 
We first define the class $\operatorname{locLip}_{\alpha}(D)$, introduced by Gehring and Martio [GM]. If $D \subset R^{n}$ is a domain and $g$ is a continuous function on $D$, we say that $g$ belongs to the class $\operatorname{locLip}_{\alpha}(D), 0<\alpha \leq 1$, if there is a constant $M<\infty$ such that

$$
|g(x)-g(y)| \leq M|x-y|^{\alpha}
$$

whenever $x, y$ lie in a ball $B$ contained in $D$. As usual, we write $g \in \operatorname{Lip}_{\alpha}(D)$ if the above inequality is valid for each pair $x, y$ of points in $D$.

Next we define Minkowski dimension which gives a measure of the size of a set in $R^{n}$ and which is analogous to Hausdorff dimension. If $E$ is a compact set in $R^{n}, 0<\delta \leq n$, and $r>0$, set

$$
M^{\delta}(E ; r)=\inf \left\{k r^{\delta}: E \subset \bigcup_{1}^{k} B(x, r)\right\} .
$$

The Minkowski content of $E$ is now

$$
M^{\delta}(E)=\limsup _{r \rightarrow 0} M^{\delta}(E, r)
$$

and the Minkowski dimension of $E$ is given by

$$
\operatorname{dim}_{M}(E)=\inf \left\{\delta: M^{\delta}(E)<\infty\right\} .
$$

Note that the Hausdorff $\operatorname{dimension}_{\operatorname{dim}_{H}}(E)$ of $E$ satisfies $\operatorname{dim}_{H}(E) \leq \operatorname{dim}_{M}(E)$; see $[\mathrm{F}]$ or $[\mathrm{MV}]$ for the relations between these two dimensions. We can now state Astala and Koskela's result [AK, Theorem 4.4].

Theorem A. Let $D^{\prime}$ be a bounded domain with

$$
\operatorname{dim}_{M}\left(\partial D^{\prime}\right)<n .
$$

If $f: D \rightarrow D^{\prime}$ is $K$-quasiconformal, then (1) and (2) are equivalent. Here $\alpha$ and $p$ depend only on each other, $n, K$, and $\operatorname{dim}_{M}\left(\partial D^{\prime}\right)$.

Our example shows that some condition like (3) is necessary in Theorem A, and, in particular, one can not replace the Minkowski dimension by the Hausdorff dimension:

Theorem B. For each $n \geq 2$ and each $K>1$ there are bounded domains $D$ and $D^{\prime}$ in $R^{n}$ quasiconformally equivalent to the unit ball of $R^{n}$ and a $K$ quasiconformal mapping $f: D \rightarrow D^{\prime}$ such that $\operatorname{dim}_{H}(\partial D)=\operatorname{dim}_{H}\left(\partial D^{\prime}\right)=n-1$ and (2) holds for all $0<\alpha<1$ but

$$
\int_{D}\left|f^{\prime}\right|^{p} d m=\infty \quad \text { for all } p>n .
$$

Astala and Koskela [AK, 5.1] also showed that if in the above situation $f$ satisfies (2) and $\alpha>\operatorname{dim}_{M}(\partial D) / n$, then (1) holds. Our second result shows that the dependence of $\alpha$ on $\operatorname{dim}_{M}(\partial D)$ in their theorem is essential. 
Theorem C. For each $n \geq 2$, each $K>1$, and each $0<\alpha<1$ there exist bounded domains $D$ and $D^{\prime}$ in $R^{n}$ quasiconformally equivalent to the unit ball of $R^{n}$ and a $K$-quasiconformal mapping $f: D \rightarrow D^{\prime}$ such that $\operatorname{dim}_{M}(\partial D)<n$ and $\operatorname{dim}_{H}(\partial D)=\operatorname{dim}_{H}\left(\partial D^{\prime}\right)=n-1$ and both (2) and (4) hold.

Still another result of Astala and Koskela [AK] states that if $\partial D$ is sufficiently smooth, say $D$ satisfies a quasihyperbolic boundary condition [GM], then (2) implies (1). Hence neither of the domains $D$ and $D^{\prime}$ in Theorem B (Theorem C respectively) can be too regular.

The basic idea behind our construction of $f, D$, and $D^{\prime}$ is the following: In order for (4) to be satisfied, we need to construct $f$ and $D$ so that $\left|f^{\prime}\right|$ will be large on a significant portion of $D$. However, if $B$ is a ball contained in $D$, then $f \in \operatorname{Lip}_{\alpha}(B)$ (we want $f$ to satisfy (2)) and hence Hölder's inequality implies that $\int_{B}\left|f^{\prime}\right| d m \leq C \operatorname{diam}(B)^{n-1+\alpha}$ where $C$ is a constant independent of $B$. Consequently, the average of $\left|f^{\prime}\right|$ on $B$ is inversely related to the size of $B$. If for each $x \in D$ we let $B_{x}$ be the largest ball satisfying $x \in B_{x} \subset D$, it follows that we must construct $D$ so that $B_{x}$ is small "most of the time". In order to accomplish this, we form $D$ by adjoining an infinite sequence of narrower and narrower projections to a rectangular domain.

We first prove Theorem C. Theorem B will then be established by a similar construction.

\section{Proof of Theorem C}

To simplify our notation, we only consider the case $n=3$ and leave the modifications needed for the general case to the reader. Let $K_{0}>1,0<\alpha<1$ and define $D_{0}=\left\{(x, y, z): 0<x<a_{0}, 0<y, z<1\right\}$; the value of $a_{0}$ is determined by the formulas (5), (10), and (11) below. Then $D$ (respectively $D^{\prime}$ ) is constructed by adjoining projections $P_{i}$ (respectively $P_{i}^{\prime}$ ) to the bottom of $D_{0}$ as pictured in Figures 1 and 2.

The projections $P_{i}$ (respectively $P_{i}^{\prime}$ ) consist of upper and lower chambers $U_{i}$ and $L_{i}$ (respectively $U_{i}^{\prime}$ and $L_{i}^{\prime}$ ). Moreover, $P_{i}$ and $P_{i}^{\prime}$ are attached to $D_{0}$ in an identical way so that $\bar{D}_{0} \cap \bar{P}_{i}=\bar{D}_{0} \cap \bar{P}_{i}^{\prime}$. The location patterns of these attachments will be defined below.

The mapping $f$ will be the identity on $D_{0}$ and map each $U_{i}$ (respectively $L_{i}$ ) onto $U_{i}^{\prime}$ (respectively $L_{i}^{\prime}$ ). On each $L_{i}, f$ will be a similarity mapping with a streching factor $s_{i}$ that increases to infinity as $i$ approaches infinity. This will allow us to deduce (4). In order to make $f K_{0}$-quasiconformal we use the regions $U_{i}$ as decompression chambers so that as one travels from $L_{i}$ to $D_{0}$ through $U_{i}$ the streching factor gradually reduces from $s_{i}$ to 1 . In order to define the subregions $U_{i}, L_{i}, U_{i}^{\prime}, L_{i}^{\prime}$ precisely, as well as give the mapping $f$ on $U_{i}$ and $L_{i}$, we fix a positive integer $i$ and translate $\bar{U}_{i} \cup \bar{L}_{i}$ and $\bar{U}_{i}^{\prime} \cup \bar{L}_{i}^{\prime}$ to $\bar{U} \cup \bar{L}$ and $\bar{U}^{\prime} \cup \bar{L}^{\prime}$ 


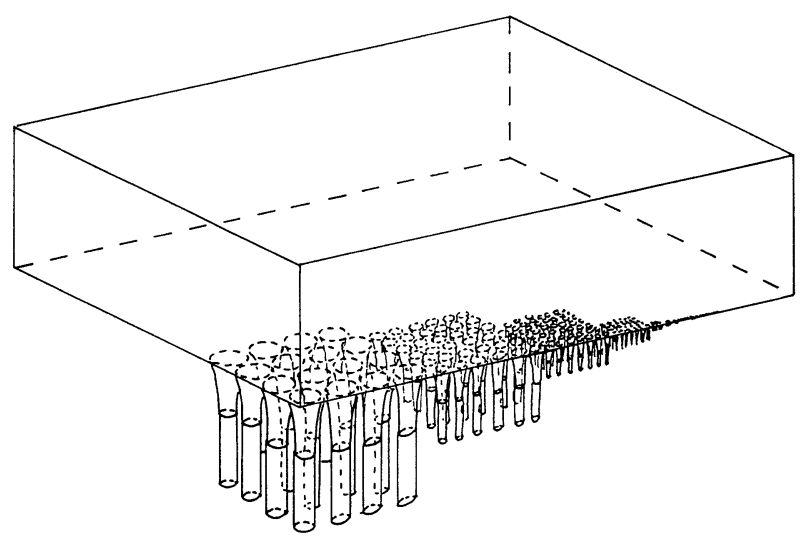

Figure 1.

so that $U \cup L$ and $U^{\prime} \cup L^{\prime}$ are symmetric with respect to the $z$-axis and $\bar{U} \cap \bar{L}$ and $\bar{U}^{\prime} \cap \bar{L}^{\prime}$ are contained in the plane $z=0$. (see Figure 2).

Here $U$ is bounded by the planes $z=0$ and $z=C r^{\gamma}$ and by the surface obtained by rotating the curve

$$
z=C\left(\frac{r^{\beta+1}}{x}-r^{\beta}\right) \frac{r^{\gamma}}{r-r^{\beta}}
$$

about the $z$-axis, $L=B(0, r) \times\left(-r^{1-\beta}, 0\right), L^{\prime}=B\left(0, r^{\beta}\right) \times(-1,0)$ and $U^{\prime}=$ $B\left(0, r^{\beta}\right) \times\left(0, C r^{\gamma-1}\left(r+r^{\beta}\right) / 2\right)$ where $B(0, s)=\left\{(x, y): x^{2}+y^{2}<s^{2}\right\}$.
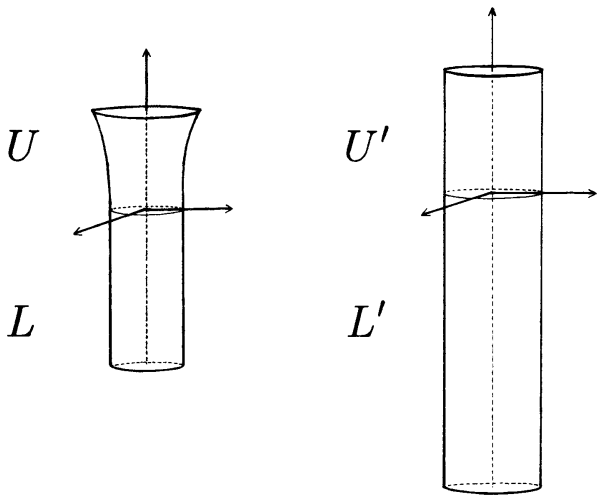

Figure 2.

Above $r=r_{i}$ is a number between 0 and 1 to be determined later, $C \geq 1$ is a constant to be determined later,

$$
\beta=\max \left\{\frac{2-\alpha}{3-2 \alpha}, \frac{4}{5}\right\}
$$


and

$$
\gamma=2 \beta-1
$$

It follows from (5), and (6) that $\gamma>0$.

We define $g$ on $\bar{U} \cup \bar{L}$ as follows

$$
g(x, y, z)= \begin{cases}\left(x w_{1}, y w_{1}, w_{2}\right), & (x, y, z) \in \bar{U} \\ \left(r^{\beta-1} x, r^{\beta-1} y, r^{\beta-1} z\right), & (x, y, z) \in \bar{L}\end{cases}
$$

where

$$
w_{1}=\frac{r-r^{\beta}}{C r^{\gamma+1}} z+r^{\beta-1}
$$

and

$$
w_{2}=\frac{r-r^{\beta}}{2 C r^{\gamma+1}} z^{2}+r^{\beta-1} z .
$$

Then it is easy to verify that $g$ is a homeomorphism of $\bar{U} \cup \bar{L}$ onto $\bar{U}^{\prime} \cup \bar{L}^{\prime}$ and $g$ is $C^{\infty}$ in $U \cup L$. We then define $f$ on $U_{i} \cup L_{i}$ by composing $g$ in an obvious way with translations so that $f$ maps $U_{i} \cup L_{i}$ onto $U_{i}^{\prime} \cup L_{i}^{\prime}$.

We next show that if we take $C$ large enough, then $g$ is $K_{0}$-quasiconformal on $U \cup L \cup\{B(0, r) \times\{0\}\}$. It obviously suffices to show that $g$ is $K_{0}$-quasiconformal on $U$. From (7) we have

$$
D g(x, y, z)=\left(\begin{array}{ccc}
w_{1} & 0 & x w_{3} \\
0 & w_{1} & y w_{3} \\
0 & 0 & w_{1}
\end{array}\right)
$$

for all $(x, y, z) \in U$; here $w_{3}=\left(r-r^{\beta}\right) /\left(C r^{\gamma+1}\right)$. Denote the operator norm of $D g(x, y, z)$ by $\|D g(x, y, z)\|$ and its determinant by $|D g(x, y, z)|$.

It follows that

$$
\begin{aligned}
\frac{\|\left. D g(x, y, z)\right|^{3}}{|D g(x, y, z)|}= & \sup _{|h|=1}\left\{\left[\left(h_{1} w_{1}+x h_{3} w_{3}\right)^{2}\right.\right. \\
& \left.\left.\quad+\left(h_{2} w_{1}+y h_{3} w_{3}\right)^{2}+{h_{3}}^{2}{w_{1}}^{2}\right]^{3 / 2} w_{1}{ }^{-3}\right\} \\
\leq & {\left[1+w_{3}{ }^{2}\left(x^{2}+y^{2}\right) w_{1}{ }^{-2}+2\left(\left|x w_{3}\right|+\left|y w_{3}\right|\right)\left|w_{1}\right|^{-1}\right]^{3 / 2} . }
\end{aligned}
$$

This last quantity is maximized for $(x, y, z) \in U$ when $|x|=|y|=r^{\beta}$ and $z=C r^{\gamma}$; so using (6) we get

$$
\begin{aligned}
\frac{\|D g(x, y, z)\|^{3}}{|D g(x, y, z)|} & \leq\left[1+2 r^{2 \beta-2 \gamma-2}\left(r-r^{\beta}\right)^{2} C^{-2}+4 r^{\beta}\left(r^{\beta}-r\right) r^{-\gamma-1} C^{-1}\right]^{3 / 2} \\
& \leq\left[1+6 C^{-1}\right]^{3 / 2}
\end{aligned}
$$


Hence, taking the constant $C$ large enough, [V, 14.3] yields $K \leq K_{0}$. It follows that $f$ is $K_{0}$-quasiconformal on $U_{i} \cup L_{i}$ and hence $K_{0}$-quasiconformal on all of $D$.

We now describe how we choose the sequence $\left\{r_{i}\right\}$ and how each $P_{i}$ (respectively $\left.P_{i}^{\prime}\right)$ is attached to $D_{0}$. For each $i$, let $E_{i}=\bar{P}_{i} \cap \bar{D}_{0}=\bar{P}_{i}^{\prime} \cap \bar{D}_{0}$. Then from the definition of $P_{i}$ and $P_{i}^{\prime}$ it follows that $E_{i}$ is a closed disk in the $z$-plane with radius $r_{i}{ }^{\beta}$. We take the $r_{i}$ 's to be positive integer powers of $\frac{1}{2}$ and for each positive integer $j$ we let $\psi(j)$ be the number of $r_{i}$ 's equal to $2^{-j}$. We arrange the corresponding $E_{i}$ 's in a square pattern $Q_{j}$ and then place the $Q_{j}$ 's so that $Q_{j}$ and $Q_{j+1}$ are adjacent and all the $Q_{j}$ 's lie along the line segment $y=0, z=0$, $0<x<a_{0}$, (see Figure 1).

If follows easily from Theorem 10.3 in [GV] that $D^{\prime}$ is quasiconformally equivalent to the unit ball of $R^{3}$, and, consequently, the same holds for $D$.

We next show that (4) is satisfied as long as $\psi$ is defined judiciously.

Let $p>3$. Since $\left|f^{\prime}\right|=r_{i}^{\beta-1}$ on $L_{i}$ and $L_{i}$ is a cylinder with dimensions $r_{i} \times r_{i}^{1-\beta}$, we have

$$
\begin{aligned}
\int_{D}\left|f^{\prime}\right|^{p} d m & \geq \sum_{1}^{\infty} \int_{L_{i}}\left|f^{\prime}\right|^{p} d m=\pi \sum_{1}^{\infty} \psi(i) 2^{-i(1-\beta)} 2^{-2 i} 2^{-i(p(\beta-1))} \\
& =\pi \sum_{1}^{\infty} \psi(i) 2^{-i((p-1)(\beta-1)+2)}
\end{aligned}
$$

On the other hand, by the definitions of $D$ and $D^{\prime}, D$ and $D^{\prime}$ will be bounded if (and only if)

$$
a_{0}=\sum_{1}^{\infty} \psi(i)^{1 / 2} 2^{-i \beta}<\infty .
$$

From (5) it follows that $\beta<1$ and hence

$$
(p-1)(\beta-1)+2=2 \beta+(p-3)(\beta-1)<2 \beta .
$$

Therefore, from (9) and (10) it follows that it is possible to choose $\psi$ so that (4) is satisfied and both $D$ and $D^{\prime}$ are bounded. For example, take

$$
\psi(i) \approx 2^{i 2 \beta} / i^{4} .
$$

Next we estimate the dimensions of $\partial D$ and $\partial D^{\prime}$.

Note first that $\partial D$ and $\partial D^{\prime}$ are both a union of a countable number of sets of finite 2-dimensional measure; hence $\operatorname{dim}_{H}(\partial D)=\operatorname{dim}_{H}\left(\partial D^{\prime}\right)=2$. Now we sketch the estimate for the Minkowski dimension of the boundary of $D$. Let $2<\delta \leq 3$, $0<r<1$, and pick an integer $i_{0}$ with $2^{-i_{0}}<r \leq 2^{-i_{0}+1}$. For each positive 
integer $i$ there are $\psi(i)$ subdomains of $D$, say $G_{i}^{j}, j=1, \ldots, \psi(i)$, corresponding to the sets $P_{i}$ constructed above with $r_{i}=2^{-i}$. Since $M^{2}\left(\partial D_{0}\right)<\infty$, we have

$$
M^{\delta}\left(\partial D \backslash \bigcup_{i} \bigcup_{j} \partial G_{i}^{j} ; r\right) \leq C_{1}
$$

where the constant $C_{1}$ is independent of $r$. Thus it suffices to consider the Minkowski content of $\cup_{i} \cup_{j} \partial G_{i}^{j}$. We estimate this content in two parts. A simple calculation which makes use of (5) and (6) shows that the 2-dimensional measure of the boundary of $G_{i}^{j}$ for each $i, j$ is comparable to $2^{i(\beta-2)}$.

By the geometry of the sets $G_{i}^{j}$ one observes that if $i \leq i_{0}$, then the surface area of the intersection of the boundary of $G_{i}^{j}$ with any ball $B$ of radius $r$ centered at the boundary of $G_{i}^{j}$ is comparable to $2^{-2 i_{0}}$. Hence a standard covering argument (e.g. using the Vitali theorem) shows that

$$
M^{\delta}\left(\bigcup_{i<i_{0}} \bigcup_{j} \partial G_{i}^{j} ; r\right) \leq C_{2} 2^{-i_{0} \delta} \sum_{i<i_{0}} \psi(i) 2^{i(\beta-2)+2 i_{0}} \leq C_{3} i_{0} 2^{i_{0}(3 \beta-\delta)},
$$

where $C_{3}$ is a constant independent of $i_{0}$. Finally, we consider the Minkowski content of the rest of the boundary of $D$. By the definition of the sets $G_{i}^{j}$, $\bigcup_{i \geq i_{0}} \bigcup_{j} G_{i}^{j}$ is contained in a rectangular region $G$ of dimensions comparable to $2^{-i_{0}(1-\beta)} \times i_{0}^{-2} \times i_{0}^{-1}$. Hence

$$
M^{\delta}\left(\bigcup_{i \geq i_{0}} \bigcup_{j} \partial G_{i}^{j} ; r\right) \leq M^{\delta}(G ; r) \leq C_{4} 2^{-i_{0} \delta} 2^{i_{0}(\beta-1)} 2^{3 i_{0}} i_{0}{ }^{-3} \leq C_{4} 2^{i_{0}(2+\beta-\delta)}
$$

here $C_{4}$ is independent of $i_{0}$. We conclude from (12), (13), and (14) that $M^{\delta}(\partial D)$ is finite provided $\delta>2+\beta$. Thus (5) yields

$$
\operatorname{dim}_{M}(\partial D) \leq 2+\max \{(2-\alpha) /(3-2 \alpha), 4 / 5\}<3
$$

as desired.

It remains to show that $f \in \operatorname{locLip}_{\alpha}(D)$. Clearly $f \in \operatorname{Lip}_{1}\left(D_{0}\right)$ and

$$
\left|f(z)-f\left(z^{\prime}\right)\right| \leq\left|z-z^{\prime}\right|^{\beta}
$$

whenever $z, z^{\prime} \in L_{i}$ satisfy $\left|z-z^{\prime}\right| \leq r_{i}$. Since $\beta \geq \alpha$, it suffices to show that $f \in \operatorname{Lip}_{\alpha}\left(U_{i}\right)$ for each positive integer $i$ with the $\operatorname{Lip}_{\alpha}\left(U_{i}\right)$ constant of $f$ independent of $i$.

Fix a positive integer $i$. We again translate $U_{i}$ and $U_{i}^{\prime}$ to $U$ and $U^{\prime}$ and work with $g$ as defined by (7). We need to show that $g \in \operatorname{Lip}_{\alpha}(U)$. Let

$$
\begin{aligned}
& g_{1}(x, y, z)=\frac{x}{r}\left(\frac{r-r^{\beta}}{C r^{\gamma}} z+r^{\beta}\right), \\
& g_{2}(x, y, z)=\frac{y}{r}\left(\frac{r-r^{\beta}}{C r^{\gamma}} z+r^{\beta}\right), \\
& g_{3}(x, y, z)=\frac{1}{r}\left(\frac{r-r^{\beta}}{2 C r^{\gamma}} z^{2}+r^{\beta} z\right) .
\end{aligned}
$$


It clearly suffices to show that $g_{1}, g_{2}$, and $g_{3}$ are each in $\operatorname{Lip}_{\alpha}(U)$.

We first note that (5) and (6) imply the following inequality

$$
\gamma \geq \frac{1-\beta}{1-\alpha}
$$

Let $\left(x_{1}, y_{1}, z_{1}\right),\left(x_{2}, y_{2}, z_{2}\right) \in U$. Then

$$
\begin{aligned}
\mid g_{3}\left(x_{1}, y_{1}, z_{1}\right)- & g_{3}\left(x_{2}, y_{2}, z_{2}\right)|=| \frac{1}{r}\left(\frac{r-r^{\beta}}{2 C r^{\gamma}}\left(z_{1}{ }^{2}-z_{2}{ }^{2}\right)+r^{\beta}\left(z_{1}-z_{2}\right)\right) \mid \\
& \leq \frac{1}{r}\left(\left|\frac{r^{\beta}}{2 C r^{\gamma}}\left(z_{1}-z_{2}\right)\left(z_{1}+z_{2}\right)\right|+r^{\beta}\left|z_{1}-z_{2}\right|\right) \leq 2 r^{\beta-1}\left|z_{1}-z_{2}\right| \\
& \leq 2 C^{1-\alpha} r^{\beta-1} r^{\gamma(1-\alpha)}\left|z_{1}-z_{2}\right|^{\alpha} \leq 2 C\left|z_{1}-z_{2}\right|^{\alpha}
\end{aligned}
$$

where the last inequality follows from (15). Consequently $g_{3} \in \operatorname{Lip}_{\alpha}(U)$.

We show that $g_{1}$ and $g_{2}$ belong to $\operatorname{Lip}_{\alpha}(U)$; by symmetry it suffices to verify that $g_{1} \in \operatorname{Lip}_{\alpha}(U)$. Observe that

$$
\begin{aligned}
& \left|g_{1}\left(x_{1}, y_{1}, z_{1}\right)-g_{1}\left(x_{2}, y_{2}, z_{2}\right)\right| \\
& \quad \leq\left|g_{1}\left(x_{1}, y_{1}, z_{1}\right)-g_{1}\left(x_{1}, y_{1}, z_{2}\right)\right|+\left|g_{1}\left(x_{1}, y_{1}, z_{2}\right)-g_{1}\left(x_{2}, y_{2}, z_{2}\right)\right| \\
& \quad=I_{1}+I_{2} .
\end{aligned}
$$

We estimate $I_{1}$ and $I_{2}$ separately. We have

$$
\begin{aligned}
I_{2} & =\left|\frac{1}{r}\left(\frac{r-r^{\beta}}{C r^{\gamma}} z_{2}+r^{\beta}\right)\left(x_{1}-x_{2}\right)\right| \leq \frac{1}{r}\left(\left|r-r^{\beta}\right|+r^{\beta}\right)\left|x_{1}-x_{2}\right| \\
& \leq 2 r^{\beta-1}\left|x_{1}-x_{2}\right| \leq 2 r^{\beta-1} 2 r^{\beta(1-\alpha)}\left|x_{1}-x_{2}\right|^{\alpha} \leq 4\left|x_{1}-x_{2}\right|^{\alpha}
\end{aligned}
$$

where the last inequality follows from (6) and (15). Estimating $I_{1}$ we get

$$
\begin{aligned}
I_{1} & =\left|x_{1} \frac{r-r^{\beta}}{C r^{\gamma+1}}\left(z_{1}-z_{2}\right)\right| \leq C^{-1} x_{1} r^{\beta-\gamma-1}\left|z_{1}-z_{2}\right| \\
& \leq C^{-1} r^{2 \beta-\gamma-1}\left|z_{1}-z_{2}\right| \leq\left|z_{1}-z_{2}\right| .
\end{aligned}
$$

From (16), (17), and (18) it follows that $g_{1} \in \operatorname{Lip}_{\alpha}(U)$.

Hence $g \in \operatorname{Lip}_{\alpha}(U)$ and the above estimates show that the corresponding constant is independent of $i$. Therefore $f \in \operatorname{Lip}_{\alpha}\left(U_{i}\right)$ with the $\operatorname{Lip}_{\alpha}\left(U_{i}\right)$ constant independent of $i$ and the proof is complete. 


\section{Proof of Theorem B}

Again, we only consider the case $n=3$. We modify the construction used to prove Theorem $\mathrm{C}$ as follows. Replace $r^{\beta}$ with $r \log (1 / r), r^{\gamma}$ with $r(\log (1 / r))^{2}$ and $r^{1-\beta}$ with $1 / \log (1 / r)$. Instead of taking the $r_{i}$ 's to be positive integer powers of $\frac{1}{2}$, the $r_{i}$ 's will now be of the form $2^{-2^{i}}$, and we denote the number of $r_{i}$ 's equal to $2^{-2^{j}}$ by $\psi(j)$. Construct $D, D^{\prime}$ and $f$ as above with these replacements.

Now

$$
\int_{D}\left|f^{\prime}\right|^{p} d m \geq \sum_{1}^{\infty} \int_{L_{i}}\left|f^{\prime}\right|^{p} d m=\pi(\log 2)^{p-1} \sum_{1}^{\infty} \psi(i) 2^{i(p-1)} 2^{-2\left(2^{i}\right)},
$$

and $D$ and $D^{\prime}$ are bounded if (and only if)

$$
\sum_{1}^{\infty} \psi(i)^{1 / 2} 2^{i} 2^{-2^{i}}<\infty
$$

Hence defining

$$
\psi(i) \approx 2^{2\left(2^{i}\right)} 2^{-2 i} i^{-4}
$$

(4) is satisfied and both $D$ and $D^{\prime}$ are bounded.

It is left to the reader to check that the mapping $f$ satisfies all the remaining requiments.

\section{References}

[AK] Astala, K., and P. Koskela: Quasiconformal mappings and global integrability of the derivative. - Mittag-Leffler Report 23, 1989/90. To appear in J. Analyse Math.

[F] Falconer, K.: Fractal geometry. - John Wiley \& Sons, Chichester, 1990.

[GM] Gehring, F.W., and O. Martio: Lipschitz classes and quasiconformal mappings. - Ann. Acad. Sci. Fenn. Ser. A I Math. 10, 1985, 203-219.

[GV] GeHrING, F.W., and J. VÄISÄLÄ: The coefficients of quasiconformality of domains in space. - Acta Math. 114, 1965, 1-70.

[MV] Martio, O., and M. Vuorinen: Whitney cubes, $p$-capacity, and Minkowski content. Exposition. Math. 5, 1987, 17-40.

[V] VÄıs̈̈L̈̈, J.: Lectures on $n$-dimensional quasiconformal mappings. - Lecture Notes in Mathematics 229. Springer Verlag, Berlin, 1971.

Bruce Hanson

St. Olaf College

Department of Mathematics

Northfield, MN 55057

U.S.A.
Pekka Koskela

University of Jyväskylä

Department of Mathematics

SF-40100 Jyväskylä

Finland

Received 4 December 1990 